\title{
First principle investigations on structural, mechanical, spin polarized electronic and magnetic properties of $\mathrm{TmZn}$ and $\mathrm{TmCd}$
}

\author{
Satish Chand ${ }^{1} \cdot$ R. P. Singh ${ }^{2}$ A. Govindan ${ }^{3}$
}

Received: 7 May 2015/Accepted: 11 August 2015/Published online: 2 September 2015

(c) The Author(s) 2015. This article is published with open access at Springerlink.com

\begin{abstract}
Structural, mechanical, spin polarized electronic and magnetic properties of $\mathrm{TmZn}$ and $\mathrm{TmCd}$ intermetallic compounds have been studied using the full-potential linearize augmented plane-wave plus local orbital method. The structural and mechanical properties have been studied in terms of lattice parameter $\left(a_{0}\right)$, bulk modulus $\left(B_{0}\right)$ and its first-order pressure derivative $\left(B_{0}^{\prime}\right)$, elastic constants $\left(C_{i j}\right)$, Young's modulus $(Y)$, shear modulus $(G)$ and Poisson's ratio $(v)$ in equilibrium state which are found to be consistent with available experimental/theoretical values. Spin polarized electronic properties have been investigated in terms of band structure and density of state histograms for spin up and spin down channel. Electronic behavior of TmZn and TmCd shows that studied materials are metallic ferromagnets with high spin polarization in which Tm-f state electrons have dominant character.
\end{abstract}

Keywords Binary intermetallics - Mechanical properties $\cdot$ Electronic structure $\cdot$ Magnetic properties

\section{Introduction}

Binary rare earth intermetallic compounds viz. TmZn and TmCd exhibit regular and systematic changes in the physical properties. Binary rare earth intermetallics offers

R. P. Singh

rishisingh79@gmail.com

1 Department of Physics, Mewar University, Chittorgarh, Rajasthan, India

2 Department of Physics, S. S. V. Degree College (Affiliated to C. C. S. University, Meerut U. P.), Hapur, India

3 Department of Physics, M. M. H. College (C. C. S. University-Meerut), Ghaziabad, India high ductility and high-temperature mechanical strength due to which they are widely used in automobile and aerospace applications, viz. commercial aircraft turbines, etc. [1-4]. Binary rare earth intermetallics are extensively studied also due to their partially filled $4 \mathrm{f}$ shells in rare earth atoms and availability of their single crystals. TmZn and $\mathrm{TmCd}$ crystallize in space group Pm3m (number 221). In Pm3m space group (221) cubic crystals, the more electronegative atoms are located at the corners of the unit cell, while the more electropositive atoms are located in the center of the unit cell [5]. The typical crystal unit cell for TmZn and TmCd is shown in Fig. 1. From this figure, it can be seen that $\mathrm{Zn}$ (or $\mathrm{Cd}$ ) sits at the center of the cubic unit cell while the rare earth atoms are at the corners.

Neutron diffraction, temperature dependence of thermoelectric power and magneto-elastic studies has been made on several rare earth intermetallic compounds [6-8]. Recently, some more studies on structural, elastic and electronic behavior have been carried out on many rare earth intermetallics [9-13]. But mechanical, spin polarized electronic and magnetic studies have not been made on $\mathrm{TmZn}$ and TmCd. Thus, these compounds have been considered for mechanical, spin polarized electronic and magnetic studies. The knowledge of mechanical, spin polarized electronic and magnetic properties of these compounds will help in further understanding and controlling the material properties which are necessary for promoting the future applications of the compounds.

\section{Computational approach}

Density functional calculations have been made to investigate the structural, mechanical, spin polarized electronic and magnetic properties of $\mathrm{TmZn}$ and $\mathrm{TmCd}$ intermetallic 


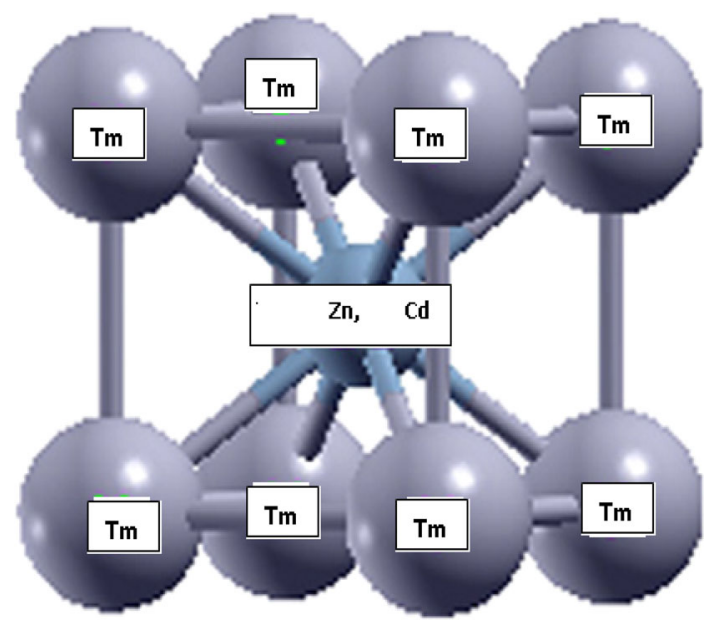

Fig. 1 Typical crystal unit cell for TmZn (or TmCd)

compounds. The calculations have been performed using an accurate full-potential linearize augmented plane-wave plus local orbital method (FP-LAPW + lo), implemented in WIEN2k package [14-18]. In this approach, the unit cell is divided into two distinct regions: (i) first region contain all points inside the muffin-tin spheres around the atom (ii) second region is the interstitial region outside the muffintin spheres. The potential and the density in the interstitial region are expanded in terms of plane waves while inside the muffin-tin sphere they are expanded in terms of spherical harmonics. Inside the muffin-tin spheres, the expansion of the non-spherical potential and charge density were carried out up to $l_{\max }=10$. The generalized gradient approximation (GGA) has been used to calculate the total energy, which is based on exchange-correlation energy optimization [15]. The $k$ and $E$ convergences were checked by increasing the number of $k$ points and the energy convergences criteria. In the irreducible part of the Brillouin zone, $14 \times 14 \times 14 k$ points were used to calculate the total and partial density of states. The lattice parameters were taken to be $3.516 \AA$ for TmZn and $3.665 \AA$ for TmCd [19] for the calculations. The radius of muffin-tin spheres was chosen to be 2.5 a.u. for Tm and 2.4 a.u. for $\mathrm{Zn}$ and Cd.

Elastic constants provide the detailed information about mechanical behavior of the materials. The elastic constants and hence other mechanical parameters viz. bulk modulus, shear modulus, Young's modulus, and Poisson's ratio can be determined by calculating the total energy of the crystal system [20, 21]:

Total energy of a crystal in strained state is given by:

$E_{\text {total }}=E_{\text {total }}^{0}+P\left(V-V_{0}\right)+\phi_{\text {elastic }}$,

where $E_{\text {total }}^{0}$ is the total energy of unstrained crystal, $V_{0}$ is the volume of the crystal in initial state, $V$ is the volume in strained state, $P$ and $\phi_{\text {elastic }}$ are the pressure and elastic energy, respectively, defined by:

$P=-\left(\frac{\partial E_{\text {total }}^{0}}{\partial V}\right)$

$\phi_{\text {elastic }}=\frac{V}{2} \cdot C_{i j k l} \varepsilon_{i k} \varepsilon_{j l}$

Here, $(i, j, k, l=1,2,3)$

In Voigt two suffix notations, $\phi_{\text {elastic }}$ is given by:

$\phi_{\text {elastic }}=\frac{V}{2} \cdot C_{i j} \varepsilon_{i} \varepsilon_{j}$,

where $C_{i j}$ are elastic moduli derived from second-order derivative of $E_{\text {total }}$ w.r.t. finite strain is given by:

$C_{i j}=\left(\frac{1}{V_{0}} \frac{\partial^{2} E_{\text {total }}}{\partial \varepsilon_{i} \partial \varepsilon_{j}}\right)_{\varepsilon=0}$.

A cubic crystal has only three independent elastic constants, namely, $C_{11}, C_{12}$ and $C_{44}$. As a result, a set of three equations is needed to determine all the constants. Hence, three types of strain must be applied to the starting crystal.

The first type strain involves calculating the bulk modulus, given by the formula

$B_{0}=\left(C_{11}+2 C_{12}\right) / 3$

Second type strain involves performing volume conservative tetragonal strain given by the following tensor:

$\left[\begin{array}{ccc}\varepsilon & 0 & 0 \\ 0 & \varepsilon & 0 \\ 0 & 0 & \frac{1}{(1+\varepsilon)^{2}}-1\end{array}\right]$.

This strain has an effect on the total energy from its unstrained state given by following equation:

$E(\varepsilon)=E(0)+3\left(C_{11}-C_{12}\right) V_{0} \varepsilon^{2}+o\left(\varepsilon^{3}\right)$

Finally, for the third type of deformation, we use the volume-conserving rhombohedral strain tensor given by

$\frac{\varepsilon}{3}\left[\begin{array}{lll}1 & 1 & 1 \\ 1 & 1 & 1 \\ 1 & 1 & 1\end{array}\right]$

This volume-conserving rhombohedral strain transforms the total energy to

$E(\varepsilon)=E(0)+\frac{1}{6}\left(C_{11}+2 C_{12}+4 C_{12}\right) V_{0} \varepsilon^{2}+o\left(\varepsilon^{3}\right)$.

Here, $O\left(\varepsilon^{3}\right)$ indicates that the neglected terms in the polynomial expansion are cubic and higher power of the $\varepsilon$.

Using the three elastic constants $C_{11}, C_{12}$ and $C_{44}$, other mechanical parameters viz. bulk modulus $(B)$ Young's modulus $(Y)$ isotropic shear modulus $(G)$ and Poisson ratio, 
Fig. 2 Total energy as a function of unit cell volume for a TmZn b TmCd with GGA approximation

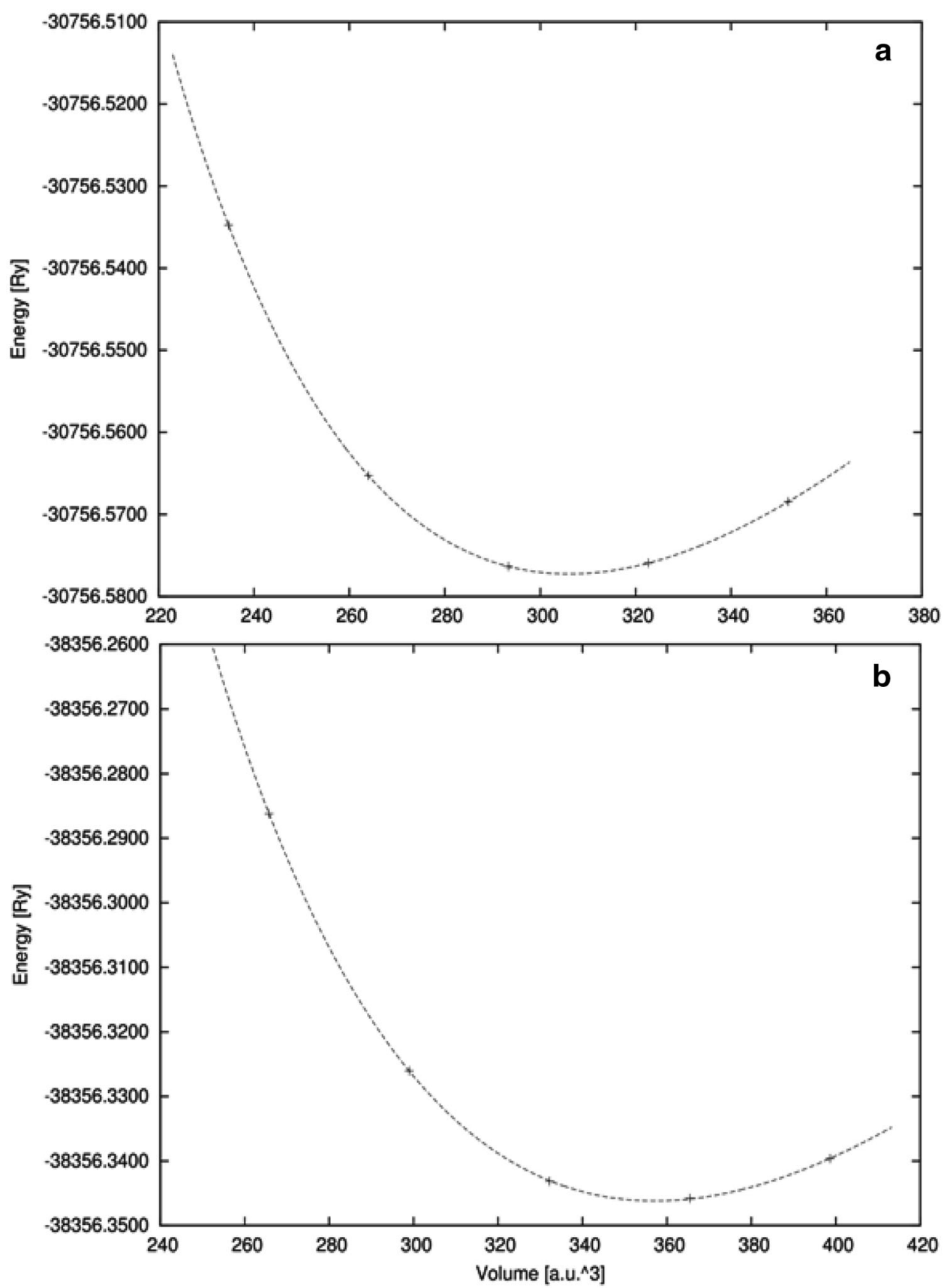

Table 1 Lattice constant, $a_{0}(\AA)$, bulk modulus, $B_{0}(\mathrm{GPa})$, pressure derivative of bulk modulus, $B_{0}^{\prime}(\mathrm{GPa})$ equilibrium condition (at $0 \mathrm{~K}$ ) for TmZn and TmCd using GGA

\begin{tabular}{llll}
\hline Compounds & $a_{0}$ & $B_{0}$ & $B_{0}^{\prime}$ \\
\hline TmZn & 3.49 & 81.23 & 4.01 \\
Expt. & $3.51^{\mathrm{a}}$ & - & - \\
TmCd & 3.63 & 54.33 & 3.54 \\
Expt. & $3.66^{\mathrm{a}}$ & - & -
\end{tabular}

${ }^{a}$ Buschow et al. [19] (v) can be calculated to show the mechanical behavior using the following explicit expressions:

$G=\left(G_{V}+G_{R}\right) / 2$.

Here $G_{\mathrm{V}}$ is Voigt's shear modulus corresponding to the upper bound of $G$ values, and $G_{\mathrm{R}}$ is Reuss's shear modulus for cubic crystals corresponding to the lower bound values, expressed as:

$$
\begin{aligned}
& G_{\mathrm{V}}=\left(C_{11}-C_{12}+2 C_{44}\right) / 4 \\
& \left.G_{\mathrm{R}}=5\left[\left(C_{11}-C_{12}\right) C_{44}\right)\right] /\left[4 C_{44}+3\left(C_{11}-C_{12}\right)\right] .
\end{aligned}
$$


Table 2 Calculated elastic moduli, $C_{i j}(\mathrm{GPa})$, bulk modulus, $B(\mathrm{GPa})$, Young's modulus, $Y(\mathrm{GPa})$, shear modulus, $G(\mathrm{GPa})$ and Poisson ratio, $v$ in equilibrium condition (at $0 \mathrm{~K}$ ) for TmZn and TmCd using GGA

\begin{tabular}{|c|c|c|c|c|c|c|c|c|c|c|}
\hline Compounds & $C_{11}$ & $C_{12}$ & $C_{44}$ & $C_{11}-C_{12}$ & $B$ & $Y$ & $G$ & $B / G$ & $v$ & $A$ \\
\hline TmZn (at $0 \mathrm{~K})$ & 117.08 & 63.37 & 56.10 & 53.44 & 81.45 & 106.71 & 41.63 & 1.95 & 0.28 & 2.10 \\
\hline Expt. (at $300 \mathrm{~K}$ ) & $103.03^{\mathrm{a}, \mathrm{c}}$ & $55.23^{\mathrm{a}, \mathrm{b}}$ & $52.20^{\mathrm{a}, \mathrm{b}}$ & $47.80^{\mathrm{a}, \mathrm{b}}$ & $70.70^{\mathrm{a}, \mathrm{b}}$ & 97.00 & 38.10 & 1.86 & 0.27 & 2.17 \\
\hline TmCd (at $0 \mathrm{~K})$ & 99.11 & 64.42 & 43.90 & 33.60 & 75.65 & 79.26 & 29.90 & 2.52 & 0.32 & 2.61 \\
\hline Expt. (at $300 \mathrm{~K}$ ) & - & - & - & $31.37^{\mathrm{a}}$ & - & - & - & - & - & - \\
\hline
\end{tabular}

${ }^{\mathrm{a}}$ Wohlfarth et al. [25], ${ }^{\mathrm{b}}$ Morin et al. [8]

And the expressions for the bulk modulus, Young's modulus and Poisson's ratio are given by:

$B=\frac{C_{11}+2 C_{12}}{3}$

$Y=\frac{9 G B}{G+3 B}$

$v=(3 B-2 G) /(6 B+2 G)$.

Elastic anisotropic factor $(A)$ is an important parameter which is the measure of anisotropy of elastic wave velocity
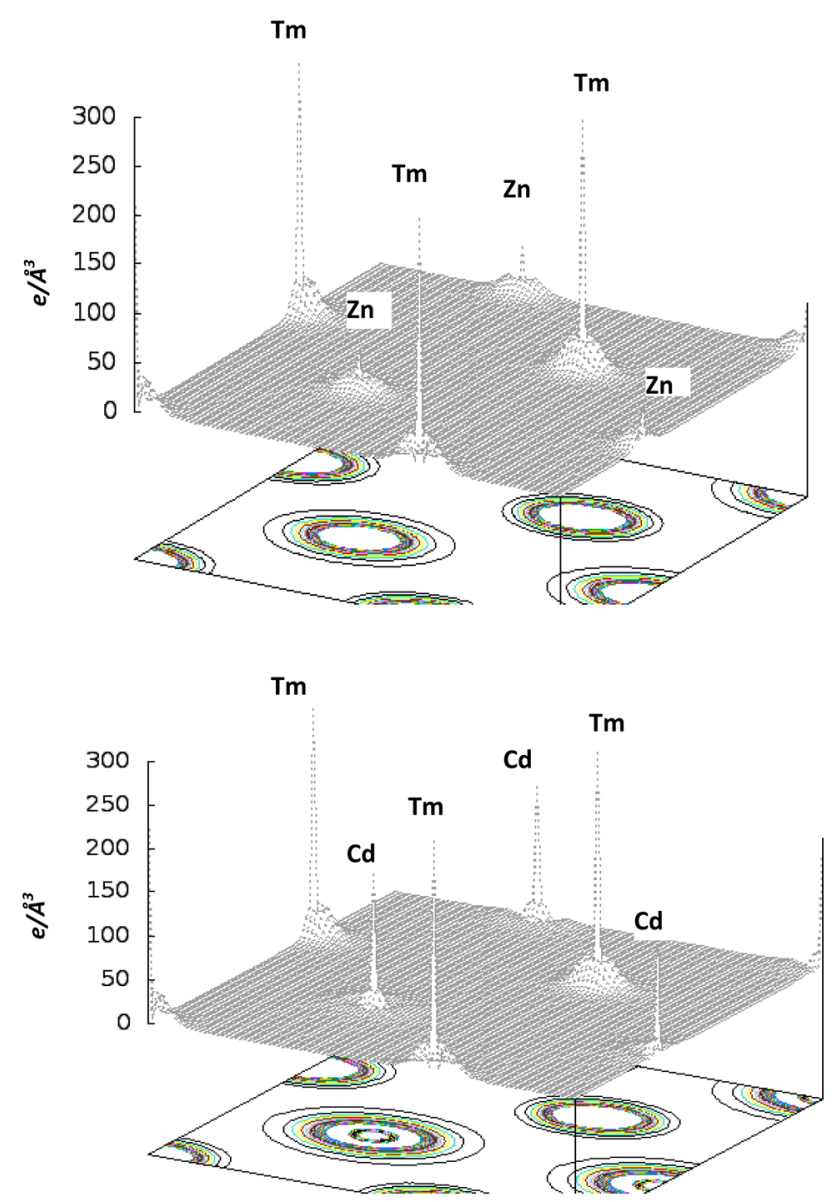

Fig. 3 3d-contour plots of the total valence charge density in (100) plane for a TmZn, b TmCd in a crystal. For an isotropic material, anisotropic factor $(A)$ is unity and expressed as [22]:

$A=\frac{2 C_{44}}{C_{11}-C_{12}}$.

\section{Results and discussion}

\section{Structural and mechanical properties}

The theoretical lattice parameters, bulk modulus and its first-order pressure derivative have been obtained through fitting the total energy data with the Murnaghan equation of state $[23,24]$ given by:

$$
\begin{aligned}
E_{\text {total }}= & E_{0}(V) \\
& +\frac{B_{0} V_{0}}{B_{0}^{\prime}\left(B_{0}^{\prime}-1\right)}\left[B_{0}\left(1-\frac{V_{0}}{V}\right)+\left(\frac{V_{0}}{V}\right)^{B_{0}^{\prime}}-1\right],
\end{aligned}
$$

where $E_{0}(V)$ and $V_{0}$ are the energy and volume at equilibrium. $B_{0}$ and $B_{0}^{\prime}$ are the equilibrium bulk modulus and its first-order pressure derivative.

The energy versus volume curves (obtained using optimization method) for $\mathrm{TmZn}$ and $\mathrm{TmCd}$ are shown in Fig. 2a, b. It is clear from these figures that equilibrium volume increases while equilibrium energy decreases from $\mathrm{TmZn} \rightarrow$ TmCd. Its reason is increasing the molecular weight from TmZn $\rightarrow$ TmCd. Because molecular stability increases with increasing the molecular weight due to which molecules vibrate in the least volume of the unit cell for occupying the equilibrium state. Hence, equilibrium unit cell volume increases and equilibrium energy decreases from $\mathrm{TmZn} \rightarrow \mathrm{TmCd}$.

The calculated lattice parameter $\left(a_{0}\right)$, bulk modulus $\left(B_{0}\right)$ and its first-order pressure derivative $\left(B_{0}^{\prime}\right)$ are shown in Table 1. Calculated values of lattice parameter show good agreement with experimental values [8,25]. Calculated values of elastic constants $\left(C_{11}, C_{12}, C_{44}\right.$ and $\left.C_{11}-C_{12}\right)$ are shown in Table 2. To best our knowledge, experimental values of elastic constants for TmZn are given in the 
Fig. 4 a Spin polarized electron dispersion curves along high symmetry directions in the Brillouin zone for a TmZn_up, b $\mathrm{TmCd} \_\mathrm{dn}$

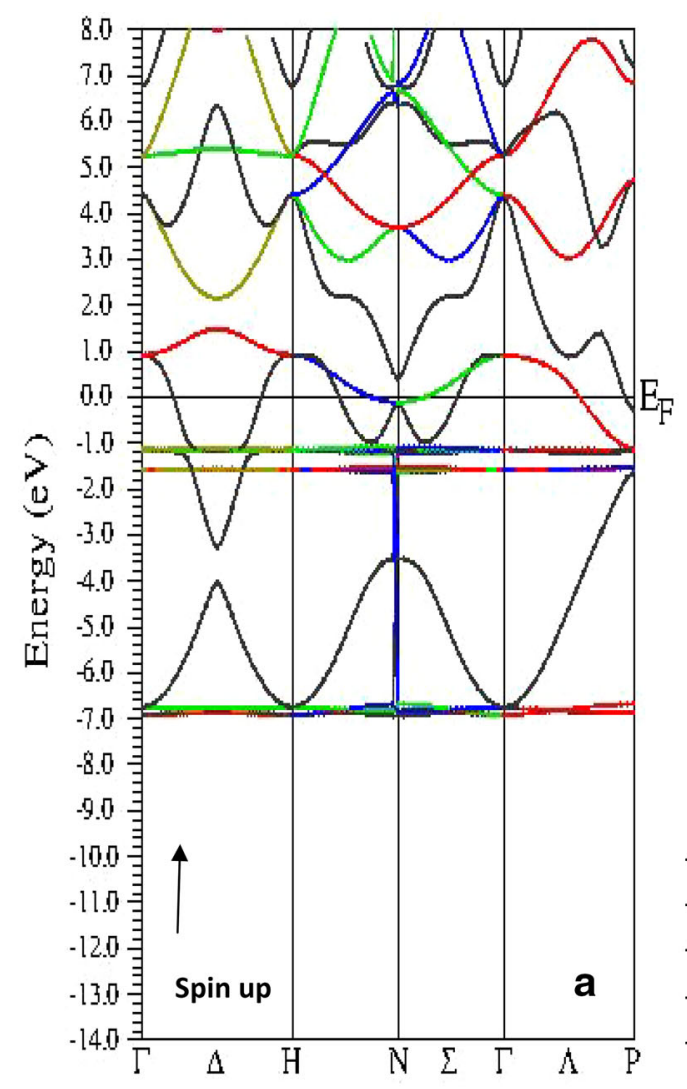

literatures which show good agreement with available experimental values. Our $C_{11}, C_{12}, C_{44}$ and $C_{11}-C_{12}$ values are found to be underestimated by about $\approx 12 \%, \approx 13 \%$, $\approx 07 \%$ and $\approx 11 \%$, respectively, for TmZn compared to the experimental value of Wohalfarath et al. [25]. Furthermore, calculated values of elastic constants $\left(C_{i j}\right)$ satisfy all mechanical stability conditions $\left(C_{11}-C_{12}>0, C_{11}>0\right.$, $\left.C_{44}>0, C_{11}+2 C_{12}>0\right)$ and cubic stability condition $\left(C_{12}<B<C_{11}\right)$ [26] that lead to the validity of calculated elastic constants.

The bulk modulus $(B)$ is a material property indicating the degree of resistance of a material to compression. Larger the bulk modulus, greater is the degree of resistance. Shear modulus, $G$ represents the resistance to plastic deformation. The calculated values of $B$ and $G$ are shown in Table 2. It can be observed from Table 2 that $B_{\mathrm{TmZn}}>B_{\mathrm{TmCd}}$ which indicates that degree of resistance of $\mathrm{TmZn}$ is larger than $\mathrm{TmCd}$. The ratio $B / G$ is a parameter which indicates the brittle or ductile behavior of the material proposed by Pugh [27]. The critical value of $B / G$ is 1.75. If $B / G>1.75$, then material indicates ductile behavior. Otherwise, the material behavior is brittle in nature. In our case, $B / G$ value for both the compounds $\mathrm{TmZn}$ and TmCd is greater than 1.75 (Table 2). Therefore, both the compounds are ductile and $\mathrm{TmCd}$ is found to be more ductile compared to TmZn. Poisson's ratio (v) also show ductile/brittle behavior of a material. If $v>0.27$, compounds are ductile otherwise brittle in nature. In our case, $v \approx 0.28$ for $\mathrm{TmZn}$ and $v \approx 0.32$ for $\mathrm{TmCd}$. Therefore, values of $v$ for $\mathrm{TmZn}$ and $\mathrm{TmCd}$ also justify that both the compounds are ductile in nature. Furthermore, Poisson's ratio also indicates the compressibility of a material. As $v \rightarrow 1 / 2$, material tends to incompressibility [28] and at $v=1 / 2$ the material is nearly incompressible. In our case, value of Poisson's ratio is $v \approx 0.28$ for $T m Z n$ and $v \approx 0.32$ for $\mathrm{TmCd}$ which show that both the compounds are compressible. The stiffness of a material is explained using Young's modulus $(Y)$ [29]. Higher value of Young's modulus indicates larger stiffness. In our case, $Y_{\mathrm{TmZn}}>$ $Y_{\mathrm{TmCd}}$, indicating $\mathrm{TmZn}$ is stiffer than $\mathrm{TmCd}$. Another important parameter is elastic anisotropic factor $(A)$ which is the measure of anisotropy of elastic wave in a crystal. For an isotropic material, $A=1$. In our case, $A>1$ indicating that both the compounds are anisotropic [22].

\section{Spin polarized electronic and magnetic properties}

Electronic charge density in terms of $3 d$-contour plots has been calculated to know the nature of chemical bonding in TmZn and TmCd which are shown in Fig. 3a, b. From Fig. 3a, b, it can be seen that there is no bonding charge to link the $\mathrm{Tm}$ and $\mathrm{Zn}$ (or $\mathrm{Cd}$ ) and charge density distribution 

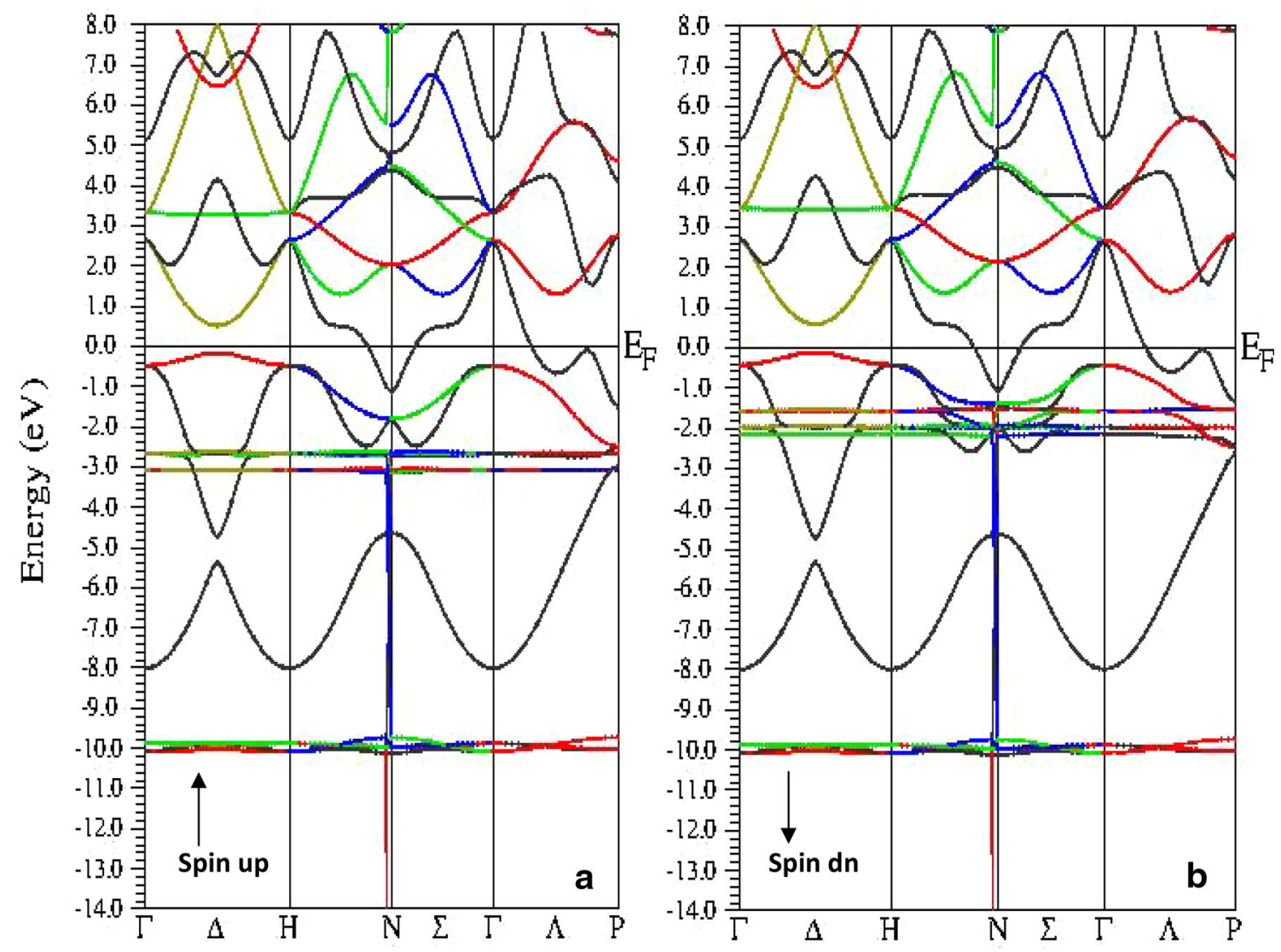

Fig. 5 a Spin polarized electron dispersion curves along high symmetry directions in the Brillouin zone for a TmZn_up, b TmCd_dn

is spherically symmetric around each atom which indicates that atoms have ionic character.

Electronic behavior of TmZn and TmCd has been shown in terms of energy bands and total, partial density of states. The calculated band structures along the high symmetry directions $\Gamma, \Delta H, \mathrm{~N}, \sum, \Lambda$ and $P$ in the Brillouin zone for spin up and spin down channel have been shown in Figs. 4a, 3b for TmZn and Fig. 5a, b for TmCd. Zero value of energy gap in the both spin up and spin down band structures indicates the metallic character, whereas zero energy gap in one band structure (either spin up or spin down) is the consequence of half metallic character of the material [30]. It can be seen from these figures that most of the valence bands lie from -7.0 to $0.0 \mathrm{eV}$ for $\mathrm{TmZn}$ and -9.0 to $0.0 \mathrm{eV}$ for TmCd (where Fermi level, $E_{\mathrm{F}}$ is considered at the origin). In both the cases (spin up and spin down configuration) and for both the compounds TmZn and $\mathrm{TmCd}$, the conduction bands are crossing the Fermi level $\left(E_{\mathrm{F}}\right)$ and overlapping significantly at the Fermi level $\left(E_{\mathrm{F}}\right)$ (i.e., zero energy gap), indicating typical conducting behavior. This behavior indicates that $\mathrm{TmZn}$ and $\mathrm{TmCd}$ exhibit the metallic character.

Figures $6 \mathrm{a}$, f and $7 \mathrm{a}-\mathrm{f}$ show the total density of states (TDOS) and partial density of states (PDOS) plots for
$\mathrm{TmZn}$ and $\mathrm{TmCd}$, respectively. In case of $\mathrm{TmZn}$, it is observed that there are three peaks in the majority spin channel below the Fermi level at around $-7.0,-1.6$ and $-1.1 \mathrm{eV}$. The peak at around $-7.0 \mathrm{eV}$ is due to mainly $\mathrm{Zn}$ $\mathrm{d}$ states (see Fig. 6e). The sharp peaks at -1.6 and $-1.1 \mathrm{eV}$ are due mainly due to hybridization of $\mathrm{Zn}-\mathrm{p}$ and Tm-f states of majority channel (see Fig. 6d, f). For spin down, three peaks were also observed in the minority spin channel below the Fermi level at around $-7.0,-0.5 \mathrm{eV}$ and third one peak is just near the Fermi level. The DOS at around $-7.0 \mathrm{eV}$ is due to $\mathrm{Zn}-\mathrm{d}$ states. DOS at around $-0.5 \mathrm{eV}$ is due to hybridization of $\mathrm{Zn}-\mathrm{p}$ and Tm-f (see Fig. 6d, f) states and DOS near the Fermi level are due to Tm-f states.

Similarly, in case of TmCd, three peaks were observed in the majority spin channel below the Fermi level at around $-9.0,-1.5$ and $-1.0 \mathrm{eV}$. The peak at around $-9.0 \mathrm{eV}$ is due to $\mathrm{Cd}-\mathrm{d}$ states (see Fig. 7e). The sharp peaks at around -1.5 and $-1.0 \mathrm{eV}$ are mainly due to hybridization of Cd-p and Tm-f states (see Fig. 7d, f). Three peaks were also observed in the minority spin channel below the Fermi level at around -9.0, $0.8 \mathrm{eV}$ and third one peak is just near the Fermi level. The sharp peak at around $-9.0 \mathrm{eV}$ is the result of $\mathrm{Cd}-\mathrm{d}$ states while the 
Fig. 6 Calculated total density of states for a TmZn; $\mathbf{b} \mathrm{Tm}$ and $\mathrm{Zn}$; c partial density of states for Tm-s, Zn-s orbital; d partial density of states for Tm-p, Zn-p orbital; e partial density of states for Tm-d, Zn-d orbital; f partial density of states for Tm-f orbital
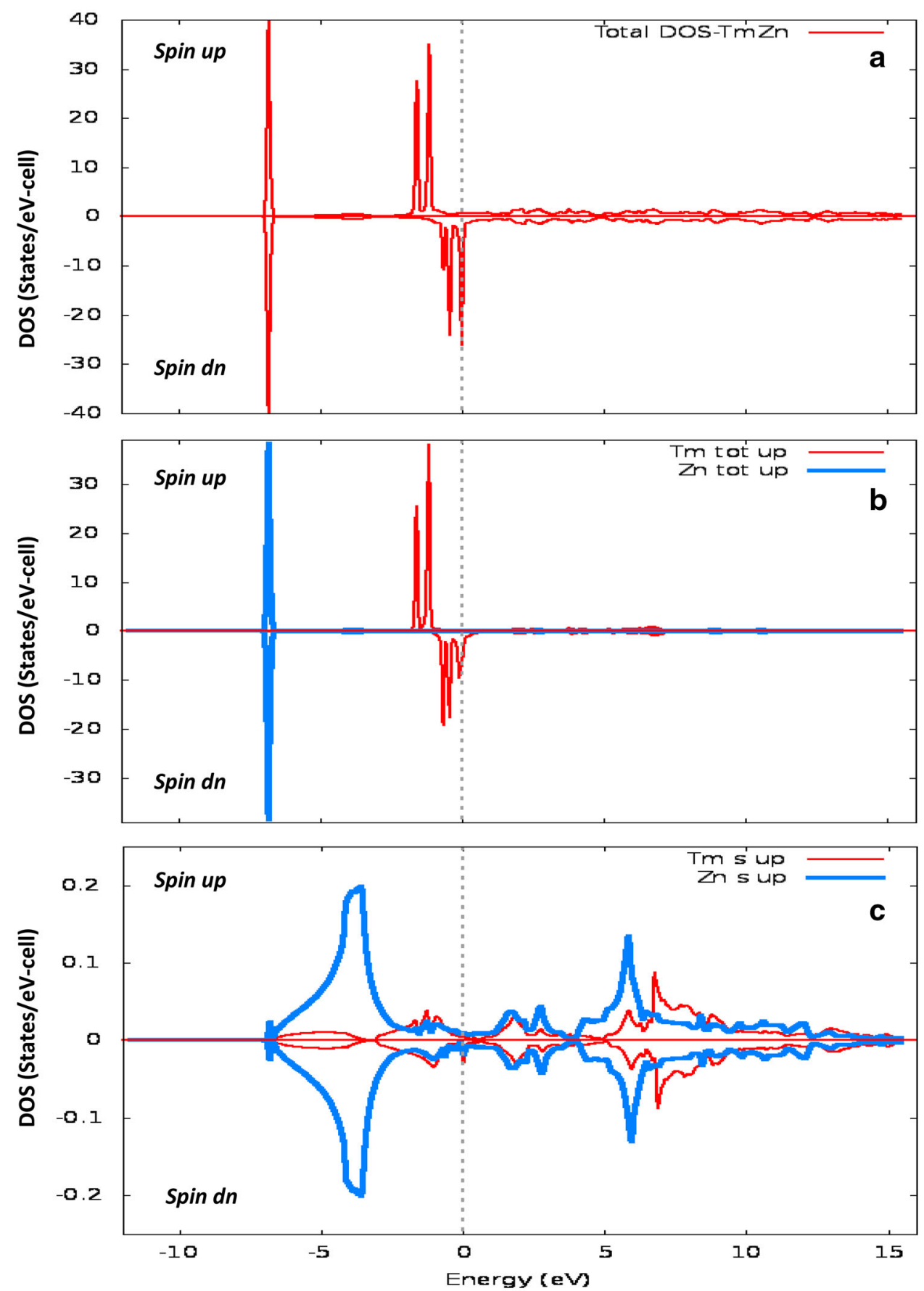

peak at around $-0.8 \mathrm{eV}$ is due to hybridization of $\mathrm{Cd}-\mathrm{p}$ and Tm-f states (see Fig. 7d, f). The sharp peak near the Fermi level is dominated by Tm-f states. It is evident from Figs. 6a-f) and $7 \mathrm{a}-\mathrm{f}$ that minority spin channel of Tm-f states are dominant near the Fermi level which is responsible for the magnetic moment. A little DOS of Zn-p (or Cd-p) are found to be empty above the Fermi level for the conduction.

The electron spin polarization at the Fermi energy $\left(E_{\mathrm{F}}\right)$ for a material can be calculated by the equation $[31,32]$ :
$P=\frac{D \uparrow\left(E_{\mathrm{F}}\right)-D \downarrow\left(E_{\mathrm{F}}\right)}{D \uparrow\left(E_{\mathrm{F}}\right)+D \downarrow\left(E_{\mathrm{F}}\right)}$.

Here, $D \uparrow\left(E_{\mathrm{F}}\right)$ and $D \downarrow\left(E_{\mathrm{F}}\right)$ are the density of states for majority and minority spin channel at the Fermi level, respectively. Here, $\uparrow$ and $\downarrow$ denote the spin up and spin down. The value of $P$ identifies the type of materials, viz. zero value of $P$ shows paramagnetic and anti-ferromagnetic character of materials even below the magnetic transition temperature. The finite value of $P$ indicates the 
Fig. 6 continued
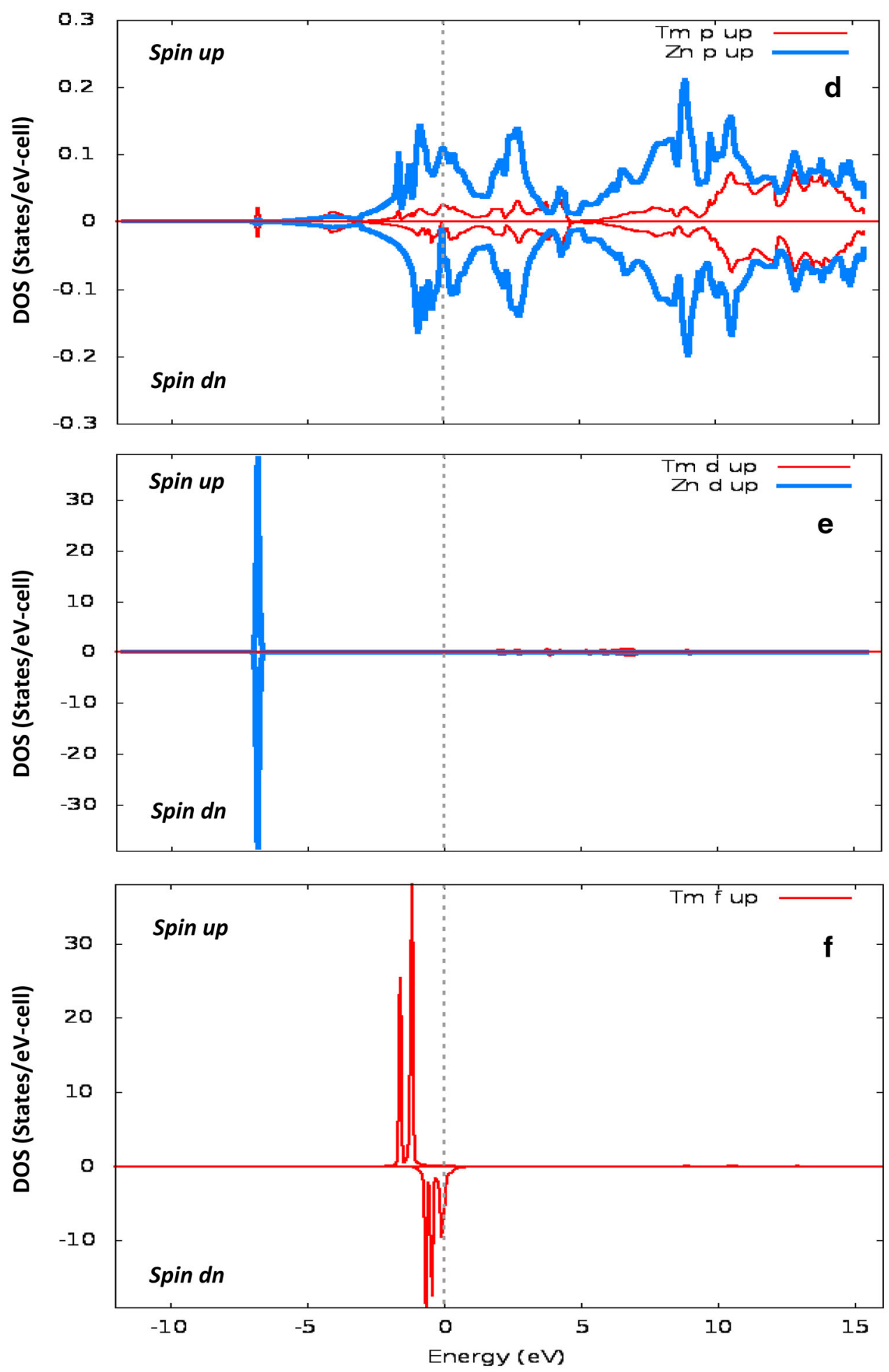

ferromagnetic material below Curie temperature [33]. Furthermore, $100 \%$ spin polarization of electrons at Fermi level is obtained when the value of $D \uparrow\left(E_{\mathrm{F}}\right)$ or $D \downarrow\left(E_{\mathrm{F}}\right)$ is zero and the material is said to be fully spin polarized.

In our both the cases, neither $D \uparrow\left(E_{\mathrm{F}}\right)$ nor $D \downarrow\left(E_{\mathrm{F}}\right)$ vanish at the Fermi level (see Fig. 6a for TmZn and Fig. 7a for TmCd) which gives finite value of $P$, implying both the compounds are typically metallic ferromagnets. The spin polarization is found to be $P \approx 92 \%$ for $\mathrm{TmZn}$ and $\approx 93 \%$ for TmCd. High percentage values of $P$ for both the compounds indicate that spin polarization is high in which mainly 4f-electrons of rare earth atom are 
Fig. 7 Calculated total density of states for a TmCd; $\mathbf{b} \mathrm{Tm}$ and $\mathrm{Cd} ; \mathbf{c}$ partial density of states for Tm-s, Cd-s orbital; d partial density of states for Tm-p, Cd-p orbital; e partial density of states for Tm-d, Cd-d orbital; f partial density of states for Tm-f orbital
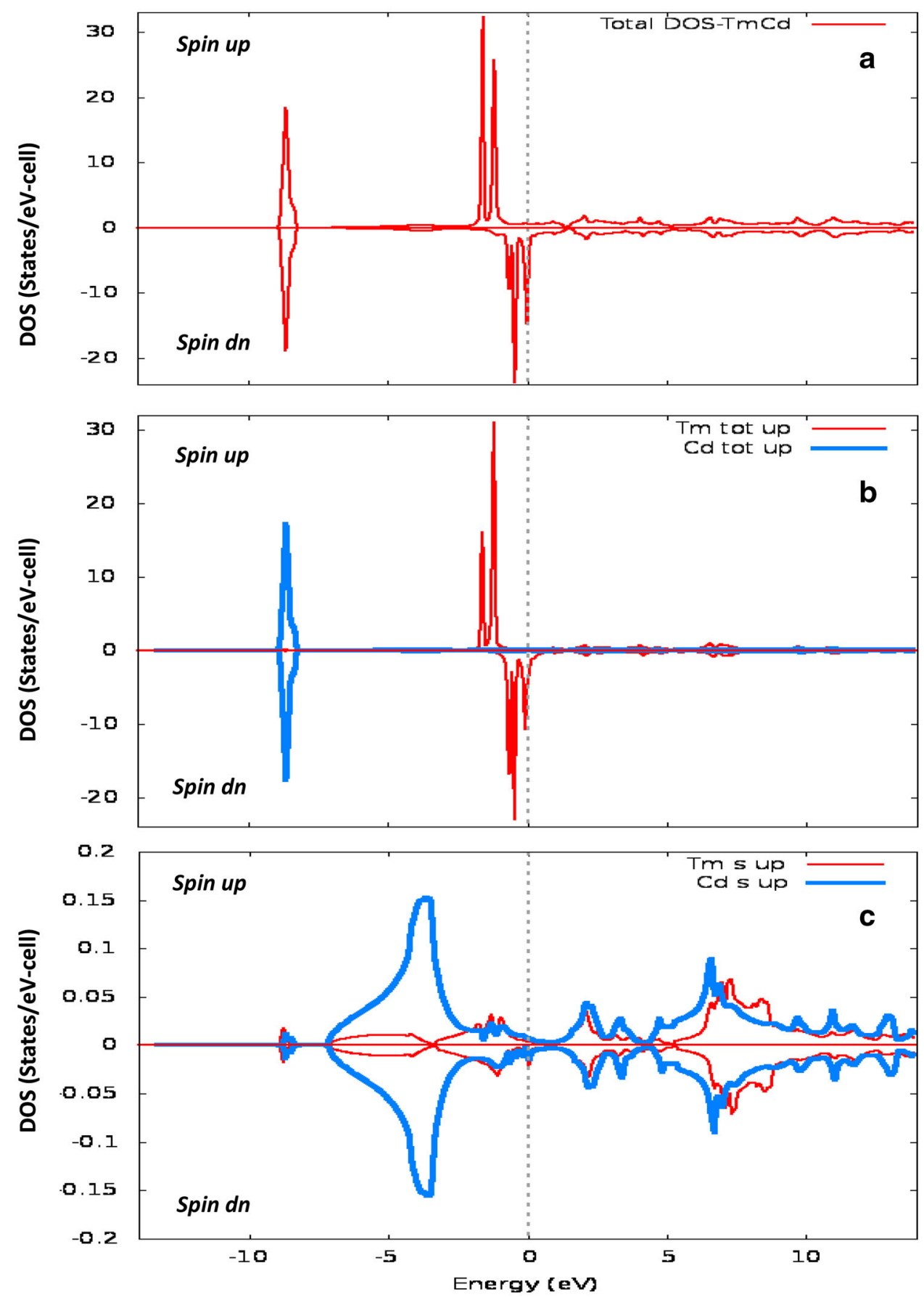

contributed mostly (see Fig. 6a, f for TmZn and Fig. 7a, f for $\mathrm{TmCd}$ ).

In the present research paper, we have performed spin polarized electronic calculations. Therefore, it is necessary to discuss about the magnetic character of these compounds. In magnetic properties, the integer value of magnetic moment is a characteristic feature of half metallic ferromagnets, whereas fractional value of magnetic moment indicates the characteristic feature of metallic ferromagnets [30]. Calculated magnetic moments on individual atoms and molecules are shown in Table 3. The total magnetic moment is found to be $1.2939 \mu_{\mathrm{B}}$ /formula unit for TmZn and $1.2961 \mu_{\mathrm{B}}$ /formula for TmCd. The calculated magnetic moments have been shown up to four digits after decimal. However, it does not mean that the magnetic moment is accurate up to four digits. The accuracy of magnetic moment calculated by WIEN2k package depends on the criteria of convergence and value of RMT which we are taking. These criteria have been used in the present study up to good accuracy. From Table 3, it can be 
Fig. 7 continued
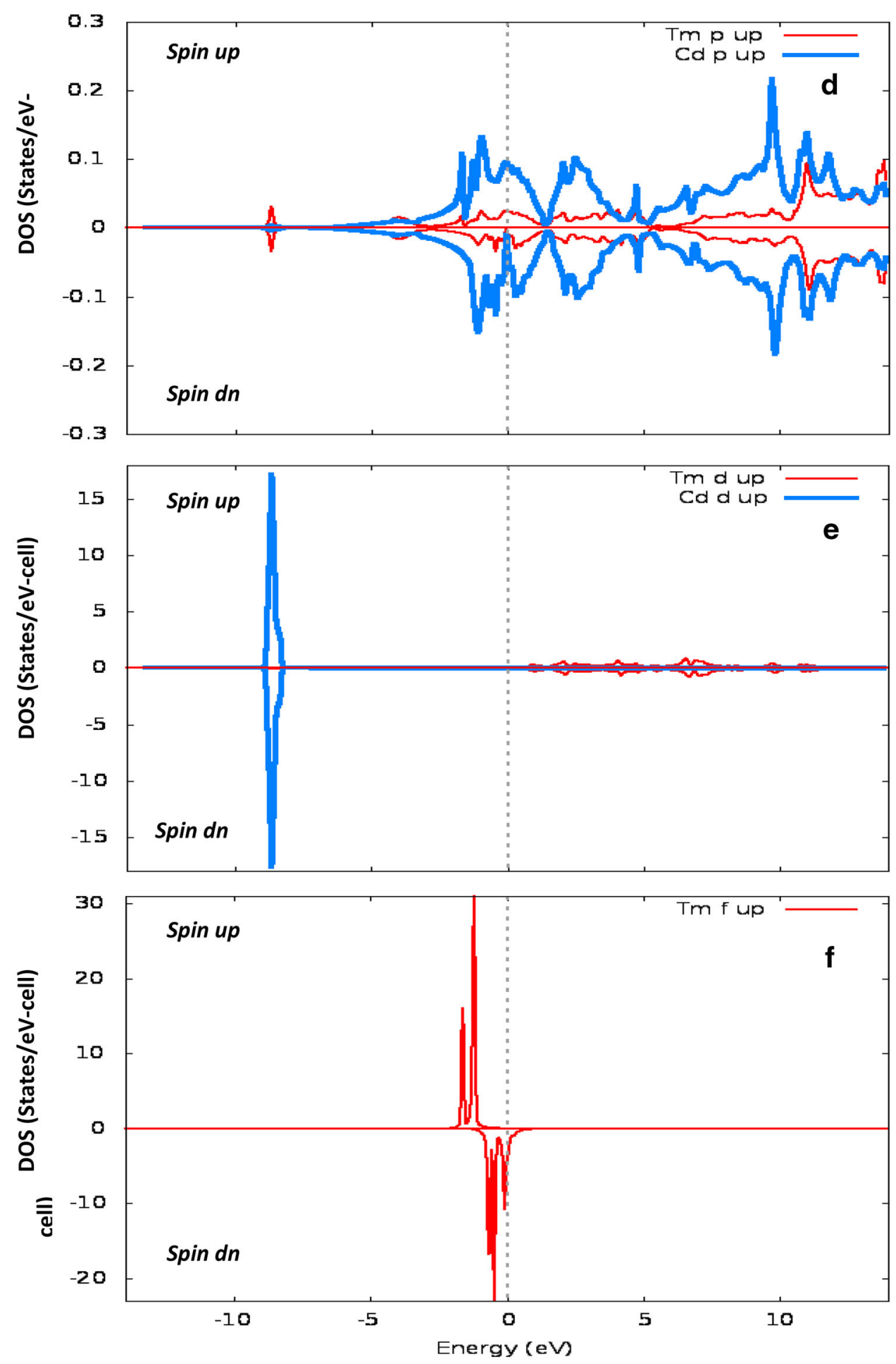

Table 3 Calculated spin magnetic moments $\left(\mu_{\mathrm{B}}\right)$ of $\mathrm{TmZn}$ and $\mathrm{TmCd}$

\begin{tabular}{llclll}
\hline & Rare earth & Interstitial region & $\mathrm{Zn}$ & $\mathrm{Cd}$ & Total magnetic moment \\
\hline $\mathrm{TmZn}$ & 1.3133 & 0.0145 & -0.00478 & - & 1.2939 \\
$\mathrm{TmCd}$ & 1.2996 & -0.0014 & - & -0.0021 & 1.2961 \\
$\mathrm{TmCu}[30]$ & 1.3626 & -0.0080 & -0.00815 & - & 1.3465 \\
\hline
\end{tabular}


seen that total magnetic moments have fractional value which favors our results of metallic ferromagnets [30]. The total magnetic moments contain three contributions: one from the rare earth atom (Tm), $\mathrm{Zn}$ (or $\mathrm{Cd}$ ) atoms and the interstitial region. $\mathrm{Zn}$ (or $\mathrm{Cd}$ ) atoms and the interstitial region are found with negligible negative magnetic moments. Thus, it is concluded that net spin magnetic moment is dominated by the $4 \mathrm{f}$-state electrons of the rare earth atom (Tm). The validation of calculated magnetic moment has been made by comparing present values with available theoretical values of magnetic moment for similar type of compounds [34]. Our calculated values match with good accuracy to similar type of compounds. Thus, our calculated values of magnetic moments are justified.

\section{Conclusions}

An accurate method named full-potential linearized augmented plane-wave plus local orbitals (FP-LAPW + lo) using generalized gradient approximation (GGA) has been used to study the structural, mechanical, spin polarized electronic and magnetic properties of $\mathrm{TmZn}$ and $\mathrm{TmCd}$ intermetallic compounds. The calculated lattice parameter and bulk modulus for $\mathrm{TmZn}$ and $\mathrm{TmCd}$ compounds are consistent with experimental/theoretical values. Calculated values of elastic constants and other mechanical parameters are found to be in good agreement with available theoretical/experimental values and indicate the ductile nature of TmZn and TmCd. 3d-contour plots and band structure histograms indicate the ionic character of $\mathrm{TmZn}$ and TmCd which is consequence of metallic character. Spin polarized electronic calculations show that studied compounds are metallic ferromagnets with high spin polarization in which mainly $4 \mathrm{f}$-electrons of Tm are taking part. Magnetic behavior of $\mathrm{TmZn}$ and $\mathrm{TmCd}$ is found to be consistent with ferromagnetic nature and the total spin magnetic moment is also dominated by $4 \mathrm{f}$-state electrons of the thulium.

Open Access This article is distributed under the terms of the Creative Commons Attribution 4.0 International License (http://crea tivecommons.org/licenses/by/4.0/), which permits unrestricted use, distribution, and reproduction in any medium, provided you give appropriate credit to the original author(s) and the source, provide a link to the Creative Commons license, and indicate if changes were made.

\section{References}

1. Gschneidner Jr, K.A., Russell, A.M., Pecharsky, A.O., Zhang, Z., Lograsso, T.A., Morris, J.R., Hsu, D.K., Lo, C.H.C., Ye, Y.Y., Slager, A.J., Kesse, D.C.: New family of ductile intermetallic compounds. Nat Mater 2, 5910 (2003)
2. Gschneidner Jr, K.A., Russell, A.M., Biner, S.B., Lo, C.H.C., Lograsso, T.A., Ye, Y., Barnard, D., Tsokol, A.O.: US Department of Energy, Basic Energy Science (2004)

3. Russell, A.M.: Ductility in intermetallic compounds. Adv. Eng. Mater. 5, 639 (2003)

4. Russell, A.M., Zhang, Z., Lograsso, T.A., Lo, C.H.C., Pecharsky, A.O., Morris, J.R., Ye, Y.Y., Gschneidner Jr, K.A., Slager, A.J.: Mechanical properties of single crystal YAg. Acta Mater. 52, 40333 (2004)

5. Gschneidner Jr, K.A., Russell, A.M., Biner, S.B., Harmon, B.N., Hsu, D.K., Lo, C.H.C., Lograsso, T.A., Pecharsky, A.O.: US Department of Energy, Basic Energy Science (2005)

6. Cable, J.W., Koehler, W.C., Wollan, E.O.: Magnetic order in rare-earth intermetallic compounds. Phys. Rev. 136(1A), A240 (1964)

7. Sousa, J.B., Minto, P.P., Amado, M.M., Moreira, I.M., Braga, M.E., Morin, P.: Temperature dependence and critical behavior of the thermoelectric power in ferromagnetic TbZn compound. J. Phys. F: Met. Phys. 10, 1809 (1980)

8. Morin, P., Williamson, S.J.: Isotropic magneto-elastic properties in thulium intermetallic compounds. Physical Review B 29, 1425 (1984)

9. Pasha, S.K., Sundareswari, M., Rajagopalan, M.: Ab initio study of the electronic structure of some B2 intermetallic compounds. Phys. B 348, 206 (2004)

10. Singh, R.P., Singh, R.K., Rajagopalan, M.: First-principle study on structural, elastic and electronic properties of rare-earth intermetallic compounds: $\mathrm{TbCu}$ and TbZn. Intermetallics 19, 1359 (2011)

11. Shugani, M., Chouhan, S.S., Aynyas, M., Sanyal, S.P.: Ab-initio study of structural, electronic and elastic properties of $\mathrm{ErCu}$. Adv. Phys. Theor. Appl. 19, 83 (2013)

12. Singh, R.P., Singh, V.K., Singh, R.K., Rajagopalan, M.: Elastic, acoustical and electronic behaviour of the RM $(\mathrm{R}=\mathrm{Dy}, \mathrm{Ho}, \mathrm{Er}$; $\mathrm{M}=\mathrm{Cu}, \mathrm{Zn}$ ) compounds. Am. J. Condens Matter Phys. 3(5), 123 (2013)

13. Pagare, G., Devi, H., Chouhan, S.S.: First principles study of electronic, elastic and thermal properties of B2 -type RECd $(\mathrm{RE}=\mathrm{La}, \mathrm{Ce}$ and $\mathrm{Pr})$ compounds. Int. J. Advance. Elect. Comp. Engg. 2(8), 248 (2013)

14. Blaha, P., Schwarz, K., Madsen, G.K.H., Kvasnicka, D., Luitz, J.: WIEN2k, An Augmented Plane Wave Plus Local Orbitals Program for Calculating Crystal Properties. University of Technology, Vienna-Austria (2001)

15. Perdew, J.P., Burke, K.: Generalized gradient approximation made simple. Phys. Rev. Lett. 77, 3865 (1996)

16. Sjostedt, E., Nordstrom, L., Singh, D.J.: An alternative way of linearizing the augmented plane wave method. Sol. Stat. Comm. 114, 15 (2000)

17. Schwarz, K., Blaha, P., Madsen, G.K.H.: Electronic structure calculations of solids using WIEN2k package for material sciences. Comput. Phys. Comm. 147, 71 (2002)

18. Monkhorst, H.J., Pack, J.D.: On special points for Brillouin zone integration. Phys. Rev. B 13, 5188 (1976)

19. Buschow, K.: Rep. Prog. Phys. 42(8), 1373 (1979). doi:10.1088/ 0034-4885/42/8/003

20. Charpin, T.: A Package for Calculating Elastic Tensors of Cubic Phase Using WIEN. Laboratory of geometrix F-75252 Paris, France (2001)

21. Shafaay, B.A., Hassan, F.E.H., Korek, M.: First principle investigation of mercury chalcogenides and their $\mathrm{HgS}_{\mathrm{x}} \mathrm{Se}_{1-\mathrm{x}}$ and $\mathrm{HgS}_{\mathrm{x}} \mathrm{Se}_{1-\mathrm{x}}$ ternary alloys. Comput. Mater. Sci. 83, 107 (2014)

22. Mayer, B., Anton, H., Bott, E., Methfessel, M., Sticht, J., Schmidt, P.C.: Ab-initio calculation of the elastic constants and thermal expansion coefficients of Laves phases. Intermetallics 11, 23 (2003) 
23. Birch, F.: Finite elastic strain of cubic crystals. Phys. Rev. 71, 809 (1947)

24. Murnaghan, F.D.: The compressibility of media under extreme pressures. Proc. Nat. Acad. Sci. USA 30, 244 (1944)

25. Wohalfarth, E.P, Buschow, K.H.J.: Handbook of Magnetic Materials 5. Elsevier, North Holland (1990)

26. Yasui, M., Terai, T., Kakeshita, T., Hagiwara, M.: Investigation of a quadrupolar interaction in $\mathrm{DyCu}$ by elastic constants measurement. J. Phys. Conf. Ser. 165, 012059 (2009)

27. Pugh, S.F.: Relations between elastic moduli and plastic properties of polycrystalline pure metals. Philos. Mag. 45, 823 (1954)

28. Mott, P.H., Dorgan, J.R., Roland, C.M.: The bulk modulus and Poisson's ratio of incompressible materials. J. Sound and Vib. 312, 572 (2008)

29. Yuan, P.F., Ding, Z.J.: Ab initio calculation of elastic properties of rock-salt and zinc-blend $\mathrm{MgS}$ under pressure. Phys. B 403, 1996 (2008)

30. Gao, G.Y., Yao, K.L., Şaşığlu, E., Sandratskii, L.M., Liu, Z.L., Jiang, J.L.: Half-metallic ferromagnetism in zinc-blende $\mathrm{CaC}$,
$\mathrm{SrC}$, and $\mathrm{BaC}$ from first principles. Phys. Rev. B 75, 174442 (2007)

31. Soulen Jr, R.J., Byers, J.M., Osofsky, M.S., Nadgorny, B., Ambrose, T., Cheng, S.F., Broussard, P.R., Tanaka, C.T., Nowak, J., Moodera, J.S., Barry, A., Coey, J.M.D: Measuring the spin polarization of a metal with a superconducting point contact. Science 282, 85 (1998)

32. Rai, D.P., Thapa, R.K.: A density functional theory study of halfmetallic ferromagnets (HMFs) behavior in $\mathrm{Co} 2 \mathrm{YSb}(\mathrm{Y}=\mathrm{Sc}, \mathrm{Ti})$. Chin. J. Phy. 51, 812 (2013)

33. Kandpal, H.C., Fecher, G.H., Felser, C.: Calculated electronic and magnetic properties of the half-metallic transition metal based Heusler compounds. J. Phys. D Appl. Phys. 40, 1507 (2006)

34. Chand, S., Singh, R.P., Govindan, A., Singh, S.K.: Mechanical, spin polarized electronic and magnetic properties of $\mathrm{TmX}$ $(\mathrm{X}=\mathrm{Cu}, \mathrm{Ag}$ ): first principle study. Int. J. Mod. Phys. B 29, 1550007 (2015) 